\title{
Diagnostic and treatment challenges in traumatic brain injury patients with severe neuropsychiatric symptoms: insights into psychiatric practice
}

This article was published in the following Dove Press journal:

Neuropsychiatric Disease and Treatment

I July 2015

Number of times this article has been viewed

\section{Margo D Lauterbach' \\ Paula L Notarangelo' \\ Stephen J Nichols ${ }^{2}$ \\ Kristy S Lane' \\ Vassilis E Koliatsos'}

'The Neuropsychiatry Program at Sheppard Pratt, Sheppard Pratt Health System, Baltimore, MD, ${ }^{2}$ Department of Emergency Medicine, The University of Tennessee College of Medicine Chattanooga, Chattanooga, TN, USA
Correspondence: Margo D Lauterbach The Neuropsychiatry Program at Sheppard Pratt, Sheppard Pratt Health System, 650I North Charles Street, PO Box 6815, Baltimore, MD 2I 285-68I5, USA

$\mathrm{Tel}+\mathrm{I} 4109384748$

Fax + I 4109385310

Email mlauterbach@sheppardpratt.org

\begin{abstract}
Traumatic brain injury (TBI) causes a variety of neuropsychiatric problems that pose diagnostic and treatment challenges for providers. In this report, we share our experience as a referral neuropsychiatry program to assist the general psychiatrist when adult TBI patients with psychiatric symptoms present for evaluation and treatment. We completed a retrospective study of patients with moderate-to-severe TBI and severe neuropsychiatric impairments. We collected information on demographics, nature of injury, symptomatology, diagnoses, and treatments. Data analysis indicates that mood stabilization was a key concern, often requiring aggressive pharmacological management. Cognitive dysfunction was a problem for the majority of patients, but was only medicated in a third, due to poor efficacy or behavioral side effects. The co-occurrence of multiple TBI-related symptoms and diagnoses in this patient cohort emphasizes the need for individualized psychopharmacological approaches and interventions.
\end{abstract}

Keywords: traumatic brain injury, neurobehavioral, treatment

\section{Introduction}

Traumatic brain injury (TBI) is a significant cause of emergency room visits, hospital stays, and morbidity and mortality in the USA. ${ }^{1}$ The leading causes of TBI are falls, motor vehicle accidents, struck by/against events including collision and contact sports, and assaults, with three peak mean ages of 0-4 years, 15-19 years, and over 65 years. ${ }^{1}$ For each group, surviving the TBI often leads to physical, behavioral/ emotional, and psychosocial changes. Specifically, a person may experience a range of difficulties including motor impairments, cognitive deficits, personality changes, and/or new or exacerbated mental illness. Psychosocial complications in the areas of employment, relationships, and social functions can be pervasive, often causing financial and family crises.

Mood, behavioral, and other psychiatric problems are very commonly encountered in the aftermath of TBI. According to one study, the adjusted relative risk of developing any psychiatric illness is several-fold higher than that in the general population in the 6 months following moderate-to-severe TBI; ${ }^{2}$ other studies show similar trends. ${ }^{3-6}$ The cause of psychiatric illness post-TBI is not always clear. In some cases, the development of a psychiatric disorder may be the direct result of the TBI; in other cases, preexisting symptoms or catastrophic changes in psychosocial functioning caused by TBI may play a primary role in the development of illness. ${ }^{7-12}$ Another major challenge in managing this unique and heterogeneous population is the lack of level-1 or level-2 evidence on therapeutic options and the paucity of treatment recommendations. ${ }^{13-16}$ 
Diagnostic systems used to categorize psychiatric illnesses, ie, the Diagnostic and Statistical Manual of Mental Disorders (DSM), fail to adequately reflect the complex neuropsychiatric presentations of patients with TBI history. DSM5 specifically mentions TBI only within the category of neurocognitive disorders ("mild" or "major" neurocognitive disorder due to TBI). Besides the former Axis I diagnoses of "idiopathic" psychotic, mood, cognitive, and anxiety disorders, TBI patients are often diagnosed with the secondary versions of all the above. Many neuropsychiatric symptoms encountered in TBI patients are similar or identical to those seen in mood, cognitive, and psychotic disorders in patients with no TBI history. ${ }^{14,17}$ The differential diagnosis between idiopathic and secondary symptoms is not always straightforward. For example, it is not easy for a clinician to ascribe the presentation of psychomotor retardation in a TBI patient to a depressive episode when it could be the direct and specific outcome of TBI. On the other hand, patients with a history of TBI may present with positive signs of psychosis but rarely show negative symptoms that are hallmarks of schizophrenia. ${ }^{14,18,19}$ The diagnosis of TBI patients who present with symptoms resembling those of "idiopathic" psychiatric illnesses but do not necessarily fulfill the required DSM criteria is a complicated matter.

Pharmacological treatment of TBI patients with neuropsychiatric presentations varies depending on the presenting symptoms. The literature is supportive of the use of psychotropic agents from several classes, including mood stabilizers, antipsychotics, and antidepressants, ${ }^{13,15,16}$ but there are essentially no major controlled trials demonstrating the efficacy of any single compound over another or guiding treatment. ${ }^{14}$

An individual with a recent or remote history of TBI often presents to the general psychiatrist for evaluation and treatment. Access to a neuropsychiatrist or TBI expert for consultation or treatment may be limited. Therefore, general psychiatrists ought to become better acquainted with the varied neuropsychiatric presentations and management options for this heterogeneous patient population. In this paper, we present retrospective data outlining our experience with the diagnosis and treatment of patients with a history of moderateto-severe TBI who attend our referral neuropsychiatry program. Because there is little published information on the diagnosis and treatment of this severely impaired psychiatric population, the present retrospective analysis was performed with the goal of helping the general psychiatrist.

\section{Methods}

We conducted a retrospective case study on patients over age 18 years who had a history of moderate-to-severe TBI and who regularly attend our neuropsychiatry program. Our program serves as a treatment referral center for TBI cases with complex neuropsychiatric problems, often co-occurring with nonpsychiatric disorders such as posttraumatic seizures. All subjects $(n=60)$ were at least 1 year post-injury and had sustained a moderate-to-severe head injury as indicated either by their original Glasgow Coma Scale score or by length of coma lasting greater than 1 day. Data were obtained from original medical records, and in some cases, additional data were solicited from parents or long-term caregivers. Causes of TBI encountered in this population were motor vehicle crashes and falls. Patients with a history of penetrating head injury were not included. Demographic information, diagnoses, presenting symptoms, and main psychotropic agents utilized at the time of chart review were recorded. Previous medication trials and premorbid psychiatric histories were available but not included in this review. Data were collected, and descriptive statistics were performed, rounding numerical data to the nearest whole number. Because this was a retrospective chart review, it was exempted from the requirement of a written informed consent by our institutional review board (IRB). Health insurance portability and accountability act (HIPAA)-compliant informed consent forms were only used when prior medical records were needed, as per IRB request.

Although all psychiatric and nonpsychiatric diagnoses were available, we focused on personality changes and mood, cognitive, and psychotic disorders. Personality changes included aggression, apathy, disinhibition, impulsivity, and hypersexuality. Cognitive syndromes included dementia, aphasia, executive dysfunction, and amnesia. Mood symptoms included depression, mania, and mood lability. Psychotic symptoms included hallucinations, delusions, and confabulatory thinking. Psychopharmacological treatments prescribed by physicians in our program were separated into non-antipsychotic mood stabilizers, antipsychotics (the overwhelming majority being second-generation agents), antidepressants, benzodiazepines, cognitive enhancers, and other.

\section{Results}

Seventy eight percent $(n=47)$ of patients included here were male, and $22 \%(n=13)$ were female. Mean age was approximately 46 years (range $23-78$ years). Although post-TBI intervals in this study ranged widely between 1 years and 44 years, the frequency of post-TBI years reveals a mode of 10 years, median of 14 years, and mean of 14.3 years. The distribution curve was skewed to the left. The majority of patients $(>50 \%)$ were seen within 15 years post-injury. There was no evidence of any relationship between post-TBI 
intervals and the nature of neuropsychiatric symptoms. Age did not appear to be related to the type of neuropsychiatric symptom encountered or pharmacological intervention utilized.

Figure 1 shows the primary and any co-occurring diagnoses used in our patient population. The vast majority of patients were diagnosed with some type of cognitive disorder such as dementia or amnestic disorder (92\%) or a personality change (88\%). A majority of patients were diagnosed with mood disorders $(67 \%)$, whereas a minority of patients were diagnosed with a psychotic disorder $(20 \%)$. Specific symptoms experienced by patients in our sample are outlined in Figure 2. Ninety five percent of patients experience cognitive dysfunction $(n=57), 87 \%(n=52)$ experience personality or behavioral changes, $68 \%(n=41)$ had symptoms of a mood disorder, $30 \%(\mathrm{n}=18)$ had psychotic symptoms, and $83 \%$ $(n=50)$ exhibited motor or sensory disturbances.
Figure 3 details all pharmacological interventions utilized for this patient population. Non-antipsychotic mood stabilizers were utilized in $80 \%$ of cases $(n=48)$, followed by antipsychotics used in $68 \%$ of cases $(n=41)$ and antidepressants in $40 \%$ of these patients $(n=24)$. Of the 40 patients with a mood disorder diagnosis, 55\% $(n=22)$ were prescribed a non-antipsychotic mood stabilizer alone, $25 \%(n=10)$ were prescribed a non-antipsychotic mood stabilizer combined with an antidepressant, and 20\% $(n=8)$ were prescribed an antidepressant alone. Ten percent of patients $(n=6)$ were prescribed two non-antipsychotic mood stabilizers, whereas $42 \%(n=25)$ were prescribed both a non-antipsychotic and an antipsychotic mood stabilizer.

For patients with impulse control problems, 20 different medications were prescribed in our sample. Mood stabilizers were prescribed to $30 \%(n=18)$ of cases followed by antipsychotics $(28 \%, n=17)$, antidepressants $(12 \%, n=7)$,

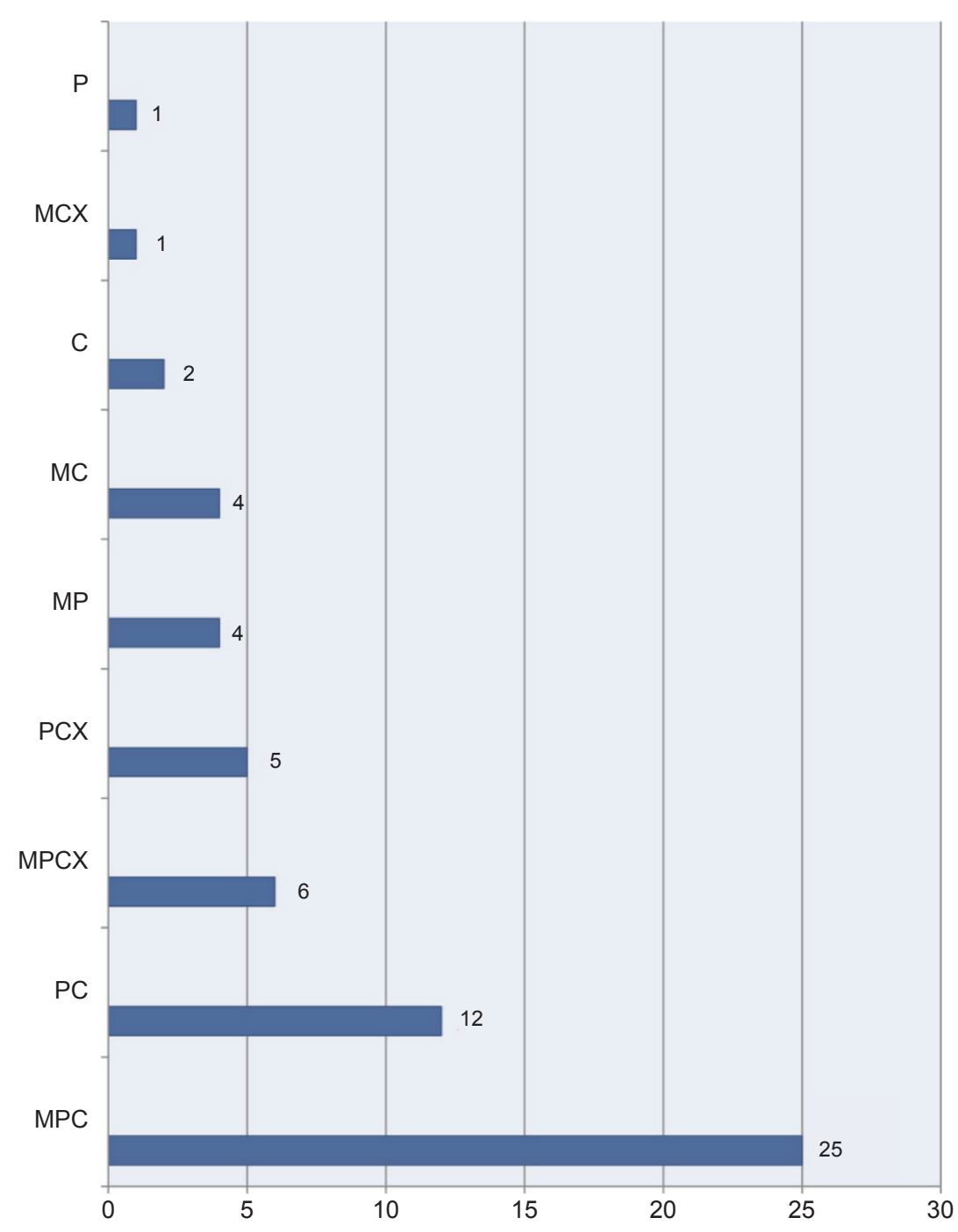

Figure I Diagnosis utilized in sample $(\mathrm{n}=60)$.

Abbreviations: $P$, personality change; $C$, cognitive; $M$, mood; $X$, psychotic. 


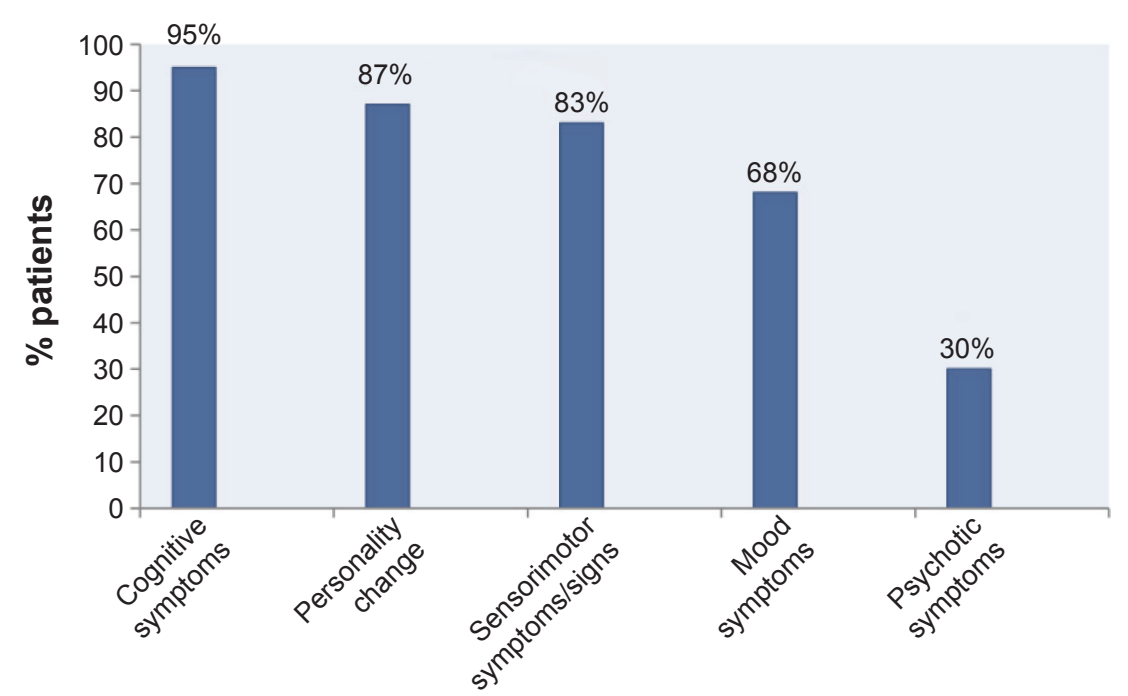

Figure 2 Frequency of neuropsychiatric symptoms $(n=60)$.

benzodiazepines $(5 \%, \mathrm{n}=3)$, medroxyprogesterone $(3 \%$, $\mathrm{n}=2)$, and beta blockers $(3 \%, \mathrm{n}=2)$. Medroxyprogesterone acetate was prescribed to two male patients to treat sexual aggression.

Thirty three percent $(n=20)$ of patients were prescribed cognitive enhancers in the form of cholinesterase inhibitors for dementia or amnestic disorders or psychostimulants targeting bradyphrenia, mental fatiguability, or attentional deficits antedating TBI. Cholinesterase inhibitors were prescribed to $20 \%$ of patients $(n=12)$, psychostimulants to $13 \%(n=8)$, modafinil to $3 \%(n=2)$, and atomoxetine was prescribed to one patient. An objective measure of cognitive function was performed at clinical appointments via serial mini-mental state examinations (MMSEs). An increase in an average of three MMSE points was found for patients after being started on anticholinesterases.

For most, unique combinations of psychotropic medications were utilized to address multiple symptoms, with $83 \%(n=50)$ requiring two or more medications. Efficacy of pharmacological interventions was determined by clinical impressions with input from patients and caregivers regarding symptom frequency and severity. Several neuropsychiatric symptoms were deemed unacceptable and led to further interventions: aggression or violence, mania, psychosis, severe insomnia with daytime somnolence or night-time wandering, poor hygiene, or social withdrawal indicative of severe depression, apathy, or cognitive decline. Thus, Table 1 highlights specifically which medication combinations

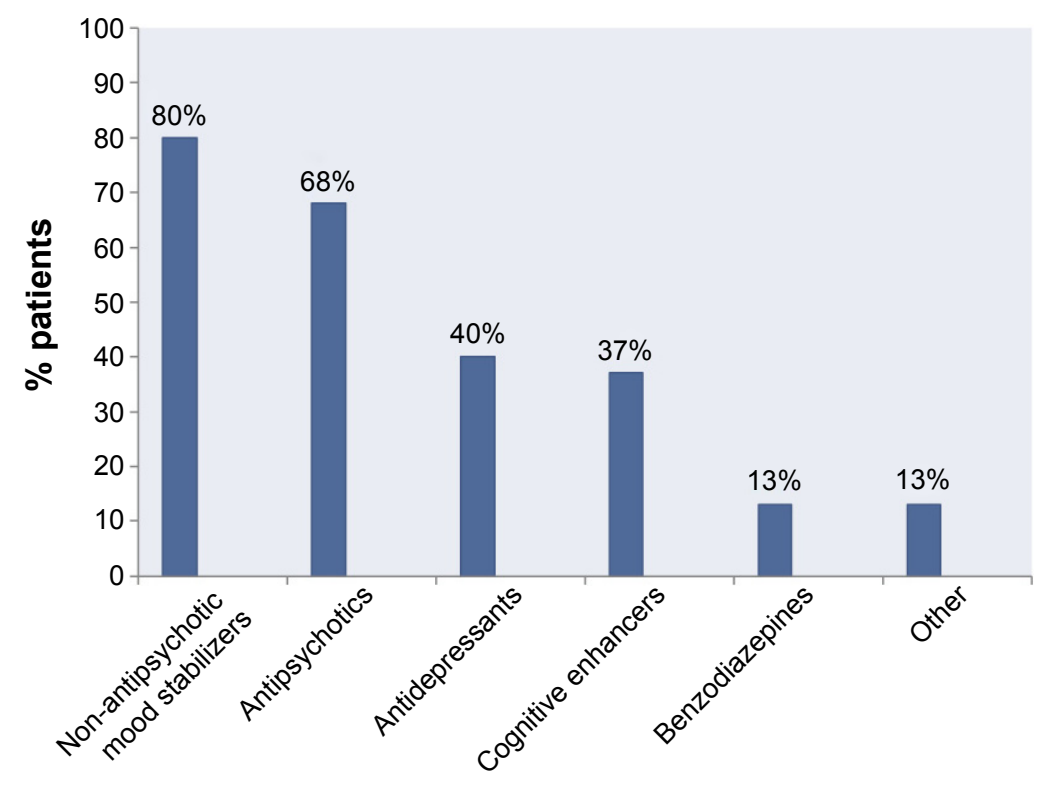

Figure 3 Pharmacological interventions $(n=60)$. 
Table I Pharmacological interventions effective for neuropsychiatric symptoms

\begin{tabular}{|c|c|}
\hline$P$ & Antipsychotics \\
\hline \multirow[t]{2}{*}{$\operatorname{MCX}$} & Non-antipsychotic mood stabilizers \\
\hline & Antipsychotics \\
\hline C & Cognitive enhancers \\
\hline \multirow[t]{3}{*}{ MC } & Non-antipsychotic mood stabilizers \\
\hline & Antidepressants \\
\hline & Cognitive enhancers \\
\hline \multirow[t]{3}{*}{ MP } & Non-antipsychotic mood stabilizers \\
\hline & Antipsychotics \\
\hline & Antidepressants \\
\hline \multirow[t]{4}{*}{ PCX } & Non-antipsychotic mood stabilizers \\
\hline & Antipsychotics \\
\hline & Antidepressants \\
\hline & Cognitive enhancers \\
\hline \multirow[t]{5}{*}{ MPCX } & Non-antipsychotic mood stabilizers \\
\hline & Antipsychotics \\
\hline & Cognitive enhancers \\
\hline & Benzodiazepines \\
\hline & Other \\
\hline \multirow[t]{5}{*}{ PC } & Non-antipsychotic mood stabilizers \\
\hline & Antipsychotics \\
\hline & Antidepressants \\
\hline & Cognitive enhancers \\
\hline & Other \\
\hline \multirow[t]{6}{*}{ MPC } & Non-antipsychotic mood stabilizers \\
\hline & Antipsychotics \\
\hline & Antidepressants \\
\hline & Cognitive enhancers \\
\hline & Benzodiazepines \\
\hline & Other \\
\hline
\end{tabular}

Abbreviations: $\mathrm{P}$, personality change; $\mathrm{C}$, cognitive; $\mathrm{M}$, mood; $\mathrm{X}$, psychotic.

were deemed effective for the major diagnostic categories (Figure 1) associated with problematic symptoms.

\section{Discussion}

Patients who suffer from severe neuropsychiatric impairments post-TBI may present to a psychiatrist with unusual diagnostic and treatment challenges. Lippert-Grüner et $\mathrm{al}^{20}$ found that psychiatric syndromes such as disinhibition and mood disorders become established by 12 months after TBI. The scarcity of evidence-based research in pharmacological interventions for these and other syndromes or symptoms postTBI presents a major challenge for treatment. The problem is further complicated in patients with severe neuropsychiatric symptoms, such as the patients reviewed in the present study. Multiple interacting factors such as premorbid psychiatric history, substance abuse, the nature, extent, and location of neuropathology, and psychosocial issues make it almost impossible to categorize these patients into uniform groups.

At least two important treatment trends were evident in the severely impaired patient population reviewed here. The first trend was that nearly $70 \%$ of these patients were diagnosed with a mood disorder and that mood stabilization was a treatment priority. The second trend was that, although cognitive impairment was diagnosed in nearly all of our patients, at the time of the study, only $33 \%$ were receiving medications to improve arousal or target symptoms such as memory deficits, bradyphrenia, or inattention.

The prevalence of mood disorders post-TBI and the importance of mood stabilization are consistent with other studies. ${ }^{21,22}$ Of course, diagnosing a patient with depression after TBI can be challenging. Certain symptoms used as diagnostic criteria for a depressive episode are not uncommon in people with a history of TBI. ${ }^{14,22}$ Psychomotor retardation, for example, could be due to major depression or be directly caused by frontal lobe damage. Similarly, a person with TBI history may manifest depressive symptoms in patterns that are different from a person who did not suffer a TBI. Depressed moods are more likely to be expressed as irritability, frustration, anger, hostility, and aggression than with sadness. ${ }^{23}$ Another challenge is ascertaining the origin of depression: depression in TBI patients can be the direct result of brain injury or evolve in the context of multiple adverse changes in relationships, employment, and finances after TBI. ${ }^{7-11,24}$ In the former scenario, Rao et a ${ }^{24}$ found a positive correlation between injury in frontal and temporal cortex or basal ganglia and symptoms of depression.

Over half of patients with a mood disorder post-TBI were treated with a mood stabilizer, either alone or combined with an antidepressant. An antidepressant alone was prescribed to only $10 \%$ of patients with a mood disorder. While commonly used antidepressants, like sertraline, are recommended in the literature for treatment of post-TBI depression, ${ }^{25} \mathrm{mood}$ stabilizers appear to be utilized more frequently in our cohort. In the majority of cases, this preference is the result of the consideration of co-occurring mood lability that often worsens with antidepressants. ${ }^{26,27}$ When mood disorders were divided into unipolar depression versus mood lability, the prescribing trends became clearer. Patients diagnosed with major depression or depressive disorder not otherwise specified (NOS) were prescribed antidepressants in nearly $80 \%$ of cases, whereas patients diagnosed with disorders featured by mood lability were prescribed mood stabilizers over $95 \%$ of the time. Given their ability to decrease agitation and mood lability, mood stabilizers can also be effective for problematic behaviors associated with personality change or cognitive dysfunction, such as impulsivity and distractibility.

The combination of two non-antipsychotic mood stabilizers was used in a little over $10 \%$ of patients. In the majority of cases, this regimen was also used to treat posttraumatic seizures or preexisting epilepsy. A little over $40 \%$ of patients were prescribed both an anticonvulsant and an antipsychotic 
mood stabilizer. For some, the mood stabilizer was intended to serve as an anticonvulsant, but for many, both classes of medications were necessary in order to control psychotic mania or aggression due to severe mood lability. Other reasons for prescribing the combination included self-injurious behaviors or frank psychosis.

As mentioned earlier, the second major trend in our patient population was a discrepancy between the nearly universal diagnosis of cognitive impairment and the pharmacological management with cognitive enhancers or stimulants of only one-third of these patients. One reason for this discrepancy is contraindications; for example, one would not prescribe stimulants to an actively aggressive patient. Other reasons are lack of efficacy or side effects; many of our TBI patients who were prescribed anticholinesterases either experienced no benefit or suffered significant worsening of aggression. Although TBI is a risk factor for dementia, ${ }^{16}$ we do not believe that the neuropsychiatric presentations in our cohort reflect late post-TBI complications such as dementia.

Symptoms of psychotic disorders after TBI are different from those seen in the general population. For example, positive symptoms of psychosis are more frequently seen than negative symptoms. ${ }^{14,18,19} \mathrm{We}$ found this pattern to be true in our patient population as well: all our patients diagnosed with psychotic disorder had positive symptoms of psychosis.

Impulsivity and sexual disinhibition were prominent in our clinical sample. These symptoms are not always easy to formulate and may be caused by a variety of factors. Impulsivity, for example, can be caused by executive (frontal lobe) dysfunction, mood lability, or be associated with attentional deficits. Accordingly, pharmacological interventions for impulsive or sexually disinhibited patients are likely to vary greatly as well: a remedy indicated for frontal impulsivity, that is, a serotonin-reuptake inhibitor, may be relatively contraindicated for impulsivity due to mood cycling.

Chronic aggression is another neuropsychiatric complication of TBI with many different facets. Aggression post-TBI can be a manifestation of disinhibition, impulsivity, and/or poor social judgment. These behavioral problems occur in patients with frontal lobe damage but can also be the result of untreated mood or psychotic disorders. The literature suggests an association between damage to the prefrontal cortex and an increase in aggressive episodes. ${ }^{21,28}$ Other studies have emphasized non-neurological factors such as decrease in social functioning, new-onset depressive disorder, or an increase in dependency for activities of daily living. ${ }^{8,9,29}$ In our patients with a mood disorder, 55\% $(n=22)$ also have chronic aggression. This trend is consistent with previously observed strong links between aggression and mood disorders. ${ }^{8,9,29}$
Beta blockers, particularly propranolol, are shown to reduce chronic aggression and are well tolerated in TBI patients. ${ }^{30}$ However, in our sample, very few patients were prescribed beta blockers. One explanation for the limited use of this intervention is the complexity of our patient population, in which aggression was often linked with mood disorders and treatment with mood stabilizers was used to address the root cause of aggression. ${ }^{31}$ In other cases, beta blockers were deemed inappropriate because of medical comorbidities, such as asthma.

The descriptive cross-sectional analysis of our patient population has several limitations. First, premorbid psychiatric diagnoses were not parsed out from new-onset diagnoses, and thus, symptoms and diagnoses in some of our patients may be related to significant premorbid psychopathology. Premorbid mood, anxiety, and substance use disorders are quite relevant when examining symptomatology, diagnostic trends, and pharmacological interventions in TBI populations. ${ }^{32}$ Second, the present study reflects medication prescribing only at the time of chart review and does not take into account previous medication trials and the reasons why medications were changed over time (ie, side effects, lack of efficacy, drug-drug interactions). Third, neuroimaging data from our patient group show some degree of neuropathological heterogeneity; this fact invites further analysis of correlations between lesion sites and diagnostic or treatment trends. Fourth, the relationship between seizures and neuropsychiatric symptoms was not established. One out of four patients in our cohort had a comorbid seizure disorder, primarily posttraumatic seizures. It is unclear as to what extent neuropsychiatric presentations in these patients were influenced by ictal, postictal, or interictal events. Finally, nonpsychiatric medications or comorbid medical disorders that may influence a person's psychiatric condition or treatment choices were not factored in our analysis. Such factors are extremely relevant for some individuals.

Several of these issues are fertile ground for further research. Sorting through these and other complexities encountered in TBI patients requires detailed analysis founded on clinical practice. It is very likely that such analyses will confirm our clinical impression that TBI patients with neuropsychiatric symptoms require a highly individualized assessment and treatment planning.

\section{Disclosure}

The authors are supported by the Leonard and Helen R. Stulman Charitable Foundation and the Women's Hospital Foundation, Inc. Dr Lauterbach is a one-third owner of Brain Educators, LLC, publishers of The Brain Card ${ }^{\circledR}$. The authors report no other conflicts of interest in this work. 


\section{References}

1. Faul M, Xu L, Wald M, et al. Traumatic Brain Injury in the United States: Emergency Department Visits, Hospitalizations and Deaths 2002-2006. Atlanta, GA: Centers for Disease Control and Prevention, National Center for Injury Prevention and Control; 2010.

2. Fann JR, Burington B, Leonetti A, Jaffe K, Katon WJ, Thompson RS. Psychiatric illness following traumatic brain injury in an adult health maintenance organization population. Arch Gen Psychiatry. 2004;61(1): 53-61.

3. Deb S, Lyons I, Koutzoukis C, Ali I, McCarthy G. Rate of psychiatric illness 1 year after traumatic brain injury. Am J Psychiatry. 1999;156(3): 374-378.

4. Hibbard MR, Uysal S, Kepler K, Bogdany J, Silver J. Axis I psychopathology in individuals with traumatic brain injury. J Head Trauma Rehabil. 1998;13(4):24-39.

5. Koponen S, Taiminen T, Portin R, et al. Axis I and II psychiatric disorders after traumatic brain injury: a 30-year follow-up study. Am J Psychiatry. 2002;159(8):1315-1321.

6. Whelan-Goodinson R, Ponsford J, Johnston L, Grant F. Psychiatric disorders following traumatic brain injury: their nature and frequency. J Head Trauma Rehabil. 2009;24(5):324-332.

7. Moldover JE, Goldberg KB, Prout MF. Depression after traumatic brain injury: a review of evidence for clinical heterogeneity. Neuropsychol Rev. 2004;14(3):143-154.

8. Rao V, Bertrand M, Rosenberg P, et al. Predictors of new-onset depression after mild traumatic brain injury. J Neuropsychiatry Clin Neurosci. 2010;22(1):100-104.

9. Jorge R, Robinson RG. Mood disorders following traumatic brain injury. Int Rev Psychiatry. 2003;15(4):317-327.

10. Hawthorne G, Gruen RL, Kaye AH. Traumatic brain injury and longterm quality of life: findings from an Australian study. J Neurotrauma. 2009;26(10):1623-1633.

11. Whitnall L, McMillan TM, Murray GD, Teasdale GM. Disability in young people and adults after head injury: 5-7 year follow up of a prospective cohort study. J Neurol Neurosurg Psychiatry. 2006;77(5): 640-645.

12. Bigler ED, Maxwell WL. Neuroimaging and neuropathology of TBI. Neuro Rehabilitation. 2011;28(2):63-74.

13. Neurobehavioral Guidelines Working Group, Warden DL, Gordon B, et al. Guidelines for the pharmacologic treatment of neurobehavioral sequelae of traumatic brain injury. J Neurotrauma. 2006;23(10):1468-1501.

14. Kim E, Lauterbach EC, Reeve A, et al; ANPA Committee on Research. Neuropsychiatric complications of traumatic brain injury: a critical review of the literature (a report by the ANPA committee on research). J Neuropsychiatry Clin Neurosci. 2007;19(2):106-127.

15. Fann JR, Hart T, Schomer KG. Treatment for depression after traumatic brain injury: a systematic review. J Neurotrauma. 2009;26(12):2383-2402.
16. Rao V, Koliatsos V, Ahmed F, Lyketsos C, Kortte K. Neuropsychiatric disturbances associated with traumatic brain injury: a practical approach to evaluation and management. Semin Neurol. 2015;35(1):64-82.

17. Himanen L, Portin R, Tenovuo O, et al. Attention and depressive symptoms in chronic phase after traumatic brain injury. Brain Inj. 2009;23(3): 220-227.

18. Guerreiro DF, Navarro R, Silva M, Carvalho M, Gois C. Psychosis secondary to traumatic brain injury. Brain Inj. 2009;23(4):358-361.

19. Arciniegas DB, Harris SN, Brousseau KM. Psychosis following traumatic brain injury. Int Rev Psychiatry. 2003;15(4):328-340.

20. Lippert-Grüner M, Kuchta J, Hellmich M, Klug N. Neurobehavioural deficits after severe traumatic brain injury (TBI). Brain Inj. 2006; 20(6):569-574.

21. Hurley RA, Taber KH. Emotional disturbances following traumatic brain injury. Curr Treat Options Neurol. 2002;4(1):59-75.

22. Perino C, Rago R, Cicolini A, Torta R, Monaco F. Mood and behavioural disorders following traumatic brain injury: clinical evaluation and pharmacological management. Brain Inj. 2001;15(2):139-148.

23. Seel RT, Macciocchi S, Kreutzer JS. Clinical considerations for the diagnosis of major depression after moderate to severe TBI. $J$ Head Trauma Rehabil. 2010;25(2):99-112.

24. Rao V, Munro CA, Rosenberg P, et al. Neuroanatomical correlates of depression in post traumatic brain injury: preliminary results of a pilot study. J Neuropsychiatry Clin Neurosci. 2010;22(2):231-235.

25. Ashman TA, Cantor JB, Gordon WA, et al. A randomized controlled trial of sertraline for the treatment of depression in persons with traumatic brain injury. Arch Phys Med Rehabil. 2009;90(5):733-740.

26. Yasseen B, Colantonio A, Ratcliff G. Prescription medication use in persons many years following traumatic brain injury. Brain Inj. 2008; 22(10):752-757.

27. Vaishnavi S, Rao V, Fann JR. Neuropsychiatric problems after traumatic brain injury: unraveling the silent epidemic. Psychosomatics. 2009; 50(3):198-205.

28. Kim E. Agitation, aggression, and disinhibition syndromes after traumatic brain injury. Neuro Rehabilitation. 2002;17(4):297-310.

29. Rao V, Rosenberg P, Bertrand M, et al. Aggression after traumatic brain injury: prevalence and correlates. $J$ Neuropsychiatry Clin Neurosci. 2009;21(4):420-429.

30. Fleminger S, Greenwood RJ, Oliver DL. Pharmacological management for agitation and aggression in people with acquired brain injury. Cochrane Database Syst Rev. 2006;4:CD003299.

31. Silver JM, McAllister TW, Yudofsky SC, editors. Textbook of Traumatic Brain Injury. 2ed ed. Washington D.C: American Psychiatric Publishing, Inc.; 2011:234.

32. Gould KR, Ponsford JL, Johnston L, Schönberger M. The nature, frequency and course of psychiatric disorders in the first year after traumatic brain injury: a prospective study. Psychol Med. 2011;41:1-11.
Neuropsychiatric Disease and Treatment

\section{Publish your work in this journal}

Neuropsychiatric Disease and Treatment is an international, peerreviewed journal of clinical therapeutics and pharmacology focusing on concise rapid reporting of clinical or pre-clinical studies on a range of neuropsychiatric and neurological disorders. This journa is indexed on PubMed Central, the 'PsycINFO' database and CAS,

\section{Dovepress}

and is the official journal of The International Neuropsychiatric Association (INA). The manuscript management system is completely online and includes a very quick and fair peer-review system, which is all easy to use. Visit http://www.dovepress.com/testimonials.php to read real quotes from published authors. 